\title{
OPEN An integrated analysis of rare CNV and exome variation in Autism Spectrum Disorder using the Infinium PsychArray
}

\author{
Elena Bacchelli ${ }^{1 *}$, Cinzia Cameli ${ }^{1,3}$, Marta Viggiano ${ }^{1,3}$, Roberta Igliozzi ${ }^{2}$, Alice Mancini ${ }^{2}$, \\ Raffaella Tancredi ${ }^{2}$, Agatino Battaglia ${ }^{2}$ \& Elena Maestrini $i^{1 *}$
}

Autism spectrum disorder (ASD) is a neurodevelopmental condition with a complex and heterogeneous genetic etiology. While a proportion of ASD risk is attributable to common variants, rare copynumber variants (CNVs) and protein-disrupting single-nucleotide variants (SNVs) have been shown to significantly contribute to ASD etiology. We analyzed a homogeneous cohort of 127 ASD Italian families genotyped with the Illumina PsychArray, to perform an integrated analysis of CNVs and SNVs and to assess their contribution to ASD risk. We observed a higher burden of rare CNVs, especially deletions, in ASD individuals versus unaffected controls. Furthermore, we identified a significant enrichment of rare CNVs intersecting ASD candidate genes reported in the SFARI database. Family-based analysis of rare SNVs genotyped by the PsychArray also indicated an increased transmission of rare SNV variants from heterozygous parents to probands, supporting a multigenic model of ASD risk with significant contributions of both variant types. Moreover, our study reinforced the evidence for a significant role of VPS13B, WWOX, CNTNAP2, RBFOX1, MACROD2, APBA2, PARK2, GPHN, and RNF113A genes in ASD susceptibility. Finally, we showed that the PsychArray, besides providing useful genotyping data in psychiatric disorders, is a valuable and cost-efficient tool for genic CNV detection, down to $10 \mathrm{~kb}$.

Autism spectrum disorders (ASDs) are a heterogeneous group of neuropsychiatric conditions characterized by impairments in social communication, as well as the presence of restricted interests, stereotyped and repetitive behaviors. ASDs have a worldwide prevalence of about $1 \%$, with males about four times more likely to be affected than females ${ }^{1}$.

ASDs are highly heterogeneous, both in clinical presentation and with reference to the complex risk architecture. ASD individuals often display other psychiatric and medical conditions including intellectual disability (ID), epilepsy, sleep disorders, motor deficits (hypotonia, apraxia or motor delay), attention-deficit hyperactivity disorder (ADHD), and gastrointestinal disturbances.

Undoubtedly, genetic factors play a substantial role in ASD risk. With the availability of microarray and massively parallel sequencing platforms, significant progresses have been made in the last decade in elucidating the underlying genetic risk factors. There is a rising awareness that both common and rare variants contribute to ASD risk. Despite the accumulating evidence supporting a major role of common genetic variation in $\mathrm{ASDs}^{2}$, the relative risk conferred by each common variant is very low, thus the identification of common risk variants robustly associated to ASD requires very large sample sizes, which are just starting to be attainable thanks to massive international efforts. A recent genome-wide association meta-analysis of 18,381 ASD cases and 27,969 controls first reported common risk variants reaching genome-wide significance ${ }^{3}$.

Given the difficulties in the identification of common risk alleles, most of our current knowledge in ASD genetics comes from the analysis of rare variants, which typically confers a much higher risk in a single individual. Large-scale genomic studies have established the role of de novo and rare inherited copy-number variants (CNVs) and protein-disrupting single-nucleotide variants (SNVs) in ASD pathogenesis. In particular, rare penetrant genic CNVs are thought to increase the risk of having ASD in 5-10\% of the individuals, depending

${ }^{1}$ Department of Pharmacy and Biotechnology, University of Bologna, Bologna, Italy. ${ }^{2}$ IRCCS Stella Maris Foundation, Viale del Tirreno 331, 56128, Calambrone, Pisa, Italy. ${ }^{3}$ These authors contributed equally: Cinzia Cameli and Marta Viggiano. *email: elena.bacchelli@unibo.it; elena.maestrini@unibo.it 
on the cohort examined. Several of these rare CNVs are recurrent and associated with other neuropsychiatric conditions, some inherited from apparently unaffected parents and none individually account for more than $1 \%$ of ASD cases, leading to the identification of hundreds of candidate genes. Particularly convincing is the role of $d e$ novo CNVs, with recent estimates that $\sim 80 \%$ of the individuals with a de novo $\mathrm{CNV}$ and an ASD diagnosis would not be affected if they did not have the $\mathrm{CNV}^{2}$, but genomic studies also suggested a role for inherited CNVs with lower penetrance ${ }^{4-6}$.

Here, we present a genome-wide CNV analysis of 127 Italian ASD families genotyped with the PsychArray, a SNP array developed by Illumina in collaboration with the Psychiatric Genomic Consortium (PGC). The PsychArray contains a genome-wide backbone of approximately 270,000 tag SNPs, 250,000 rare and low-frequency exonic variants, and approximately 50,000 custom markers selected based on evidence from prior genetic studies of psychiatric disorders, including ASD. Here we show that, despite the rather low genome-wide SNP density, the PsychArray allows a reliable detection of genic CNVs down to $10 \mathrm{~kb}$. In addition, the exome and rare variant content of this SNP array allows the identification of an interesting subset of rare genic variants. The main aim of our study was thus to conduct an integrated analysis of both CNV and SNV data in a clinically well-defined collection of ASD Italian families.

At present, whole genome "chromosomal microarray" (CMA) is recommended as a first tier clinical genetic test for detecting disease-causing CNVs in individuals with ASD or other neurodevelopmental disorders $^{7-9}$. However, it can sometimes be difficult to establish or exclude the clinical relevance of specific $\mathrm{CNVs}$, as most CNVs are characterized by incomplete penetrance and variable expressivity. Accumulation of genotype-phenotype information is therefore essential to consolidate known ASD loci and to identify novel candidate genes, with the aim of expanding our knowledge of ASD's genetic background and improving the clinical care of people with ASD. A secondary aim of our study was thus to provide detailed clinical data of individuals carrying known causal CNVs and/or variants.

\section{Results}

Rare CNV burden analysis. The characteristics of our clinical sample are reported in Table 1. All DNA samples, including 128 ASD individuals from 127 pedigrees, 238 parents and 365 controls were genotyped using the Illumina Infinium ${ }^{\circledR}$ PsychArray (Fig. 1).

To our knowledge, only a few studies reported the use of PsychArray data for genome-wide CNV detection $^{10-12}$. Hence, we established a CNV calling protocol based on three different CNV detection algorithms and set criteria to define stringent CNV calls (Supplementary Methods). To test the reliability of our protocol we tested 31 CNVs for validation using qPCR (Supplementary Table S1 and Supplementary Fig. S1). All tested CNV were validated, demonstrating the specificity of our algorithm of this cost-effective array in detecting small genic CNVs, down to approximately $10 \mathrm{~Kb}$. To test the sensitivity of the PsychArray for genic CNV detection and our analysis protocol, we have included an ASD sample with a 15q13.3 duplication, spanning a region of approximately $500 \mathrm{~kb}$ including the entire CHRNA7 gene, that was previously genotyped with the high-density array Illumina $1 \mathrm{M}$-duo array ${ }^{5}$. As expected, the PsychArray wasn't able to detect most intragenic CNVs and CNVs spanning segmental duplication regions called by the Illumina $1 \mathrm{M}$-duo array, due to the lack of probes in those regions; conversely, it allowed the identification of all rare genic CNVs called by the $1 \mathrm{M}$-duo array, including the clinically relevant CHRNA7 duplication (see Supplementary Table S2).

According to our stringent criteria, a total of 253 rare CNVs ( $<1 \%$ frequency) were detected among $128 \mathrm{ASD}$ cases, while 639 rare CNVs were identified in 363 controls (Supplementary Table S3).

To test the impact of rare CNVs in cases and controls, we performed a global CNV burden analysis. As shown in Table 2, there is a higher proportion of cases who have at least one rare CNV event compared to controls $(0.90$ versus 0.82 , empirical $p=0.019)$, and this difference is more significant if we consider only deletions $(0.66$ versus 0.54 , empirical $p=0.008$ ). Moreover, there is a trend for an increased rate of rare CNVs in ASD cases compared to controls, and this increased rate becomes significant when only deletions are considered (1.05 versus 0.81 , empirical $p=0.009$ ).

To investigate if CNVs affecting genes previously associated with ASD mainly contribute to the observed burden results, we used the SFARI gene database (https://gene.sfari.org/) and its ranking system, restricting the analysis to the set of SFARI ASD genes (Supplementary Table S4). The number of SFARI genes affected by deletions and the proportion of subjects having at least one event are significantly higher in cases versus controls (Table 2). Notably, re-analysis of the deletion burden after removal of those deletions affecting SFARI genes lacked significance in both comparisons (GRATE $p=0.18$, GPROP $p=0.16$ ).

Finally to confirm the presence of a statistically significant enrichment for SFARI ASD candidate genes, we performed gene-set enrichment analysis controlling for case-control differences in CNV rate and size, showing a significant enrichment for the count of ASD candidate gene affected by a CNV (empirical $p=0.034$ ) in cases versus controls.

CNVs overlapping with known genomic disorders loci. In order to identify in our cohort CNVs of potential clinical significance, we sought CNV overlapping loci previously implicated in known genomic disorders (https://decipher.sanger.ac.uk/) and recurrent CNV shown to increase the risk of developing early-onset neurodevelopmental disorders ${ }^{13}$.

We identified 5 CNVs (2 deletions and 3 duplications) in regions known to associate with autosomal genomic disorders, most of which correspond to genomic hotspots flanked by segmental duplication (Table 3a). The two identified deletions are both de novo, while the 3 duplications are inherited from unaffected parents.

The female proband $\mathrm{AB} 133$ has a terminal de novo deletion involving the distal part of chromosome 22q13.33 that includes the SHANK3 gene, considered the culprit gene for the Phelan-McDermid syndrome (PMS). PMS associated deletions vary in size from 45 kilobases $(\mathrm{kb})$ to more than nine megabases $(\mathrm{Mb})$ with a possible 


\begin{tabular}{|c|c|c|c|c|c|c|c|c|c|c|c|c|}
\hline & \multirow[b]{2}{*}{$n$} & \multicolumn{3}{|c|}{ All sample $(n=128)$} & \multirow[b]{2}{*}{$n$} & \multicolumn{3}{|c|}{ Males $(n=106)$} & \multirow[b]{2}{*}{$n$} & \multicolumn{3}{|c|}{ Females $(n=22)$} \\
\hline & & Mean & SD & Range & & Mean & SD & Range & & Mean & SD & Range \\
\hline \multicolumn{13}{|l|}{ ADI-R (diagnosis) $)^{a}$} \\
\hline Autism $(3 / 3)^{\mathrm{b}}$ & 48 & - & - & - & 35 & - & - & - & 13 & - & - & - \\
\hline Not autism $(2 / 3)^{c}$ & 7 & - & - & - & 7 & - & - & - & 0 & - & - & - \\
\hline \multicolumn{13}{|l|}{ ADI-R (score) ${ }^{\mathrm{a}}$} \\
\hline Social interaction & 55 & 17.5 & 5.0 & $8-28$ & 42 & 17.5 & 5.3 & $8-28$ & 13 & 17.5 & 4.1 & $12-25$ \\
\hline Communication and Language & 55 & 11.8 & 4.6 & $5-23$ & 42 & 12.0 & 4.4 & $5-23$ & 13 & 11.4 & 5.4 & $7-22$ \\
\hline $\begin{array}{l}\text { Restricted and repetitive } \\
\text { behaviours }\end{array}$ & 55 & 5.9 & 2.3 & $2-12$ & 42 & 6.0 & 2.4 & $2-12$ & 13 & 5.3 & 2.0 & $3-9$ \\
\hline \multicolumn{13}{|l|}{ ADOS (diagnosis) $^{\mathrm{d}}$} \\
\hline Autism $^{\mathrm{e}}$ & 56 & - & - & - & 43 & - & - & - & 13 & - & - & - \\
\hline Autism Spectrum $^{\mathrm{f}}$ & 30 & - & - & - & 26 & - & - & - & 4 & - & - & - \\
\hline Non spectrum ${ }^{g}$ & 6 & - & - & - & 4 & - & - & - & 2 & - & - & - \\
\hline \multicolumn{13}{|l|}{$\operatorname{ADOS}$ (score) $^{\mathrm{d}}$} \\
\hline Communication domain & 83 & 4.5 & 1.9 & $0-11$ & 65 & 4.4 & 1.9 & $0-11$ & 18 & 4.7 & 2.2 & $1-8$ \\
\hline Social domain & 83 & 8.2 & 2.9 & $3-14$ & 65 & 8.1 & 2.7 & $3-14$ & 18 & 8.6 & 3.7 & $4-14$ \\
\hline Social plus communication & 88 & 12.7 & 4.2 & $5-22$ & 70 & 12.6 & 3.9 & $5-22$ & 18 & 13.3 & 5.6 & $6-22$ \\
\hline Stereotypic behavior & 68 & 2.4 & 1.8 & $0-7$ & 55 & 2.2 & 1.6 & $0-7$ & 13 & 3.2 & 2.4 & $0-6$ \\
\hline Play & 64 & 2.2 & 1.4 & $0-6$ & 50 & 2.2 & 1.4 & $0-6$ & 14 & 2.6 & 1.2 & $0-4$ \\
\hline \multicolumn{13}{|l|}{ Language level (ADOS module) ${ }^{\mathrm{h}}$} \\
\hline $\begin{array}{l}\text { non verbal- single/some words } \\
(\bmod 1)\end{array}$ & 57 & - & - & - & 44 & - & - & - & 13 & - & - & - \\
\hline $\begin{array}{l}\text { phrase or fluent speech }(\bmod \\
2 / 3 / 4)\end{array}$ & 31 & - & - & - & 26 & - & - & - & 5 & - & - & - \\
\hline \multicolumn{13}{|l|}{ VABS (age equivalents) $^{i}$} \\
\hline $\begin{array}{l}\text { Age at VABS administration } \\
\text { (months) }\end{array}$ & 108 & 80.10 & 35.90 & $27-186$ & 87 & 81.70 & 36.28 & $27-186$ & 21 & 73.48 & 34.62 & $37-160$ \\
\hline $\begin{array}{l}\text { Communication domain } \\
\text { (months) }\end{array}$ & 104 & 45.05 & 34.48 & $18-147$ & 83 & 47.37 & 35.21 & $18-147$ & 21 & 35.86 & 30.50 & $18-142$ \\
\hline Receptive (months) & 105 & 33.05 & 30.08 & $18-194$ & 84 & 35.08 & 32.27 & $18-194$ & 21 & 24.90 & 17.15 & $18-81$ \\
\hline Expressive (months) & 104 & 41.08 & 30.70 & $18-120$ & 83 & 43.00 & 31.33 & $18-120$ & 21 & 33.48 & 27.43 & $18-120$ \\
\hline Written (months) & 105 & 55.81 & 28.93 & $37-157$ & 84 & 57.55 & 29.60 & $37-157$ & 21 & 48.86 & 25.58 & $37-140$ \\
\hline $\begin{array}{l}\text { Daily living Skills domain } \\
\text { (months) }\end{array}$ & 107 & 42.74 & 29.45 & $18-175$ & 86 & 43.97 & 28.42 & $18-168$ & 21 & 37.71 & 33.64 & $18-175$ \\
\hline Personal (months) & 107 & 38.80 & 25.22 & $18-133$ & 86 & 39.65 & 25.29 & $18-133$ & 21 & 35.33 & 25.21 & $18-124$ \\
\hline Domestic (months) & 104 & 56.98 & 33.26 & $34-203$ & 83 & 58.05 & 32.41 & $34-203$ & 21 & 52.76 & 36.96 & $34-203$ \\
\hline Community (months) & 105 & 42.81 & 26.96 & $26-168$ & 84 & 44.42 & 27.34 & $26-168$ & 21 & 36.38 & 24.94 & $27-141$ \\
\hline Socialization domain (months) & 106 & 31.65 & 17.87 & $18-130$ & 85 & 33.58 & 19.20 & $18-130$ & 21 & 23.86 & 6.98 & $18-37$ \\
\hline $\begin{array}{l}\text { Interpersonal relationship } \\
\text { (months) }\end{array}$ & 103 & 28.80 & 18.03 & $18-145$ & 82 & 29.56 & 18.65 & $18-145$ & 21 & 25.81 & 15.40 & $18-83$ \\
\hline Play and Leisure time (months) & 104 & 32.41 & 22.86 & $18-141$ & 83 & 33.46 & 21.87 & $18-121$ & 21 & 28.29 & 26.60 & $18-141$ \\
\hline Coping skills (months) & 103 & 45.00 & 18.33 & $18-125$ & 83 & 45.77 & 17.88 & $18-122$ & 20 & 41.80 & 20.24 & $32-125$ \\
\hline Motor skills domain (months) & 97 & 41.91 & 14.34 & $18-65$ & 78 & 43.62 & 13.88 & $18-65$ & 19 & 34.9 & 14.4 & $18-65$ \\
\hline Gross Motor (months) & 98 & 43.60 & 14.44 & $18-64$ & 79 & 44.84 & 14.12 & $18-64$ & 19 & 38.47 & 14.99 & $18-64$ \\
\hline Fine Motor (months) & 97 & 40.59 & 16.07 & $18-66$ & 78 & 42.73 & 15.79 & $18-66$ & 19 & 31.79 & 14.50 & $18-66$ \\
\hline \multicolumn{13}{|l|}{ Intelligence Quotient (IQ) ${ }^{\mathfrak{j}}$} \\
\hline Total IQ ${ }^{\mathrm{k}}$ & 34 & 78.5 & 19.1 & $36-112$ & 31 & 77.7 & 19.0 & $49-112$ & 3 & 86.3 & 22.5 & $70-112$ \\
\hline Verbal IQ ${ }^{1}$ & 35 & 81.0 & 20.0 & $43-124$ & 31 & 79.6 & 20.0 & $47-124$ & 4 & 91.8 & 19.3 & 75-109 \\
\hline Non Verbal IQ ${ }^{\mathrm{m}}$ & 75 & 84.6 & 22.9 & $31-135$ & 63 & 86.0 & 22.5 & $39-135$ & 12 & 77.3 & 24.7 & $31-113$ \\
\hline \multicolumn{13}{|l|}{ Developmental level (GMDS) $^{\mathrm{n}}$} \\
\hline $\begin{array}{l}\text { Global Developmental Quotient } \\
\text { (DQ) }\end{array}$ & 20 & 57.8 & 17.0 & $31-79$ & 17 & 59.6 & 17.4 & $31-79$ & 3 & 47.3 & 11.7 & $37-60$ \\
\hline A - Locomotor & 11 & 77.1 & 16.9 & $45-109$ & 10 & 78.2 & 17.4 & $45-109$ & 1 & 66.0 & - & - \\
\hline B - Personal-Social & 12 & 52.6 & 15.0 & $28-77$ & 9 & 58.1 & 12.5 & $31-77$ & 3 & 36.0 & 7.5 & $28-43$ \\
\hline $\mathrm{C}$ - Hearing and language & 23 & 40.5 & 18.9 & $10-73$ & 19 & 44.2 & 18.4 & $17-73$ & 4 & 23.0 & 9.8 & $10-31$ \\
\hline D - Eye and Hand Co-ordination & 13 & 56.2 & 23.8 & $28-96$ & 10 & 62.2 & 23.8 & $29-96$ & 3 & 36.0 & 7.5 & $28-43$ \\
\hline E - Performance & 29 & 66.2 & 22.9 & $29-107$ & 23 & 69.5 & 22.4 & $29-107$ & 6 & 53.5 & 21.9 & $36-93$ \\
\hline F - Practical Reasoning & 2 & 67.5 & 17.7 & $55-80$ & 2 & 67.5 & 17.7 & $55-80$ & 0 & - & - & - \\
\hline \multicolumn{13}{|l|}{ Child Behavior Checklist (t-scores) } \\
\hline Continued & & & & & & & & & & & & \\
\hline
\end{tabular}




\begin{tabular}{|c|c|c|c|c|c|c|c|c|c|c|c|c|}
\hline & \multirow[b]{2}{*}{$n$} & \multicolumn{3}{|c|}{ All sample $(n=128)$} & \multirow[b]{2}{*}{$n$} & \multicolumn{3}{|c|}{ Males $(n=106)$} & \multirow[b]{2}{*}{$n$} & \multicolumn{3}{|c|}{ Females $(n=22)$} \\
\hline & & Mean & SD & Range & & Mean & SD & Range & & Mean & SD & Range \\
\hline Internalize problems & 97 & 63.8 & 8.8 & $45-86$ & 79 & 63.1 & 8.9 & $45-86$ & 18 & 66.7 & 7.5 & $49-78$ \\
\hline Externalize problems & 97 & 57.8 & 9.7 & $33-77$ & 79 & 57.6 & 9.9 & $33-77$ & 18 & 58.6 & 8.6 & $44-74$ \\
\hline Total problems & 97 & 63.2 & 9.7 & $38-89$ & 79 & 62.6 & 9.9 & $38-89$ & 18 & 65.5 & 8.4 & $46-77$ \\
\hline
\end{tabular}

Table 1. Summary of the clinical and diagnostic characteristics of the ASD sample. ${ }^{\text {a Autism Diagnostic }}$ Interview - Revised; ' Probands met all 3 criteria for an ASD diagnosis based on ADI-R; 'Probands 2/3 criteria for ASD diagnosis based on ADI-R: they have a diagnosis of ASD based on the other assessments and/or

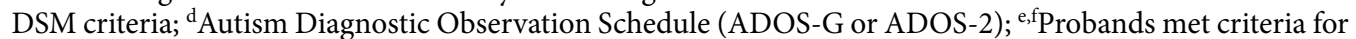
a diagnosis of autism or autism spectrum based on ADOS; 'PProbands did not meet criteria for an autism/ASD diagnosis based on ADOS: they have a diagnosis of ASD based on the other assessments and/or DSM criteria;

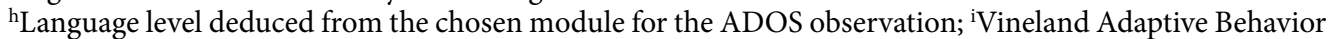

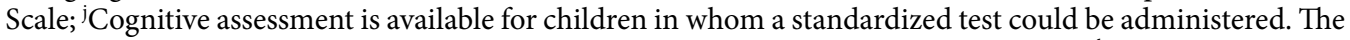
test choice was based on age, language level and individual characteristics of the subject; ${ }^{k}$ Average of the total IQ scores obtained from the assessments of children who were given WPPSI-III, WISC (R, III or IV), KBIT-2 scales; ${ }^{1}$ Average of the verbal IQ scores obtained from the assessments of children who were given WPPSIIII, WISC (R, III or IV), KBIT-2 scales; ${ }^{\mathrm{m}}$ Average of the non verbal IQ scores obtained from the assessments of children who were given WPPSI-III, WISC (R, III or IV), KBIT-2 or Leiter-R scales; ${ }^{\mathrm{n}}$ Griffiths Mental Development Scales.

correlation between deletion size and presence and/or severity of some PMS symptoms ${ }^{14}$. The de novo 22q13.33 deletion identified in our ASD proband, presenting with absence of speech and ID, spans only about $530 \mathrm{~kb}$.

A de novo $645 \mathrm{~kb}$ interstitial deletion encompassing the $1 \mathrm{p} 36.32$ locus was identified in the male proband AB27. Microdeletion 1p36 is a well characterized microdeletion syndrome associated with ID of variable degree and other clinical features, such as epilepsy and characteristic craniofacial features ${ }^{15}$. Interstitial deletions are present in about $29 \%$ of the patients, while approximately $52 \%$ have a de novo terminal $1 \mathrm{p} 36$ deletion ${ }^{16}$. The deletion reported here spans 9 genes from TNFRSF14-AS1 to the first 2 coding exons of the PRDM16 gene, and is among the smallest reported in the 1 p36 locus. Our proband, with a high functioning ASD, had lax ligaments, deep-set eyes, and bilateral overfolded helix; but none of the distinctive clinical features of the "deletion 1p36" syndrome. Such findings confirm that the critical region for the core phenotype of the syndrome is toward the distal end of the short arm of chromosome 1 (1p36.33-1p36.32).

The male proband AB164, with ASD /ADHD, and borderline cognitive impairment, without dysmorphic features, carries a duplication on chromosome 17q12, inherited from an apparently healthy mother, overlapping the $17 \mathrm{q} 12$ recurrent duplication. This is consistent with the observation that, in approximately $90 \%$ of cases, the $17 q 12$ duplication is inherited from a parent who is often minimally affected or phenotypically normal ${ }^{17}$.

A paternal inherited duplication encompassing the region 16p13.11 is present in the female proband AB35, with ASD, and mild cognitive impairment, without dysmorphic features, which overlaps with the likely core pathogenic region at the $16 \mathrm{p} 13.11$ locus (interval II) and includes the NDE1 gene, which is the major candidate gene for the neurodevelopmental phenotypes associated with the $16 \mathrm{p} 13.11 \mathrm{CNVs}^{18}$.

Finally, a 15q11.2 duplication, overlapping the BP1-BP2 genomic interval was identified in male proband AB111, with ASD, mild cognitive impairment, without dysmorphic features. The BP1-BP2 CNVs has been shown to have a modest impact on ASD risk, however maternal duplications transmitted to male probands, as in our case, have been suggested to confer a greater effect on ASD-related phenotype ${ }^{19}$.

De novo CNVs. Using the trio-based CNV calling algorithm implemented in PennCNV, and subsequent qPCR validation we identified 4 rare genic de novo CNVs: the two de novo deletions described above (Table $3 \mathrm{a}$ ), and two additional de novo CNVs that are smaller, do not overlap known loci, and therefore have a more uncertain pathogenic role (Table $3 b$ ).

Case AB161, with ASD and borderline cognitive impairment, without dysmorphic features, has a de novo duplication on chromosome 5p13.2 including 4 genes, namely CAPSL, IL7R, SPEF2, UGT3A1. SPEF2 is entirely duplicated; it encodes for Sperm flagellar protein 2, involved in cilia function ${ }^{20}$ and its transcript is a FMRP target ${ }^{21}$. Proband AB161 displayed two other inherited rare genic CNVs: a maternal deletion of 3 genes on chr16p13.3, and a paternal duplication on chromosome 1q43 including two genes: ACTN2 and MTR. Interestingly, the MTR gene is another FMRP target gene and it has been implicated in methylcobalamin deficiency type G (MIM:250940), an autosomal recessive inherited disease that causes mental retardation, macrocytic anemia, and homocystinuria.

Male proband AB84, with ASD and a relative macrocephaly, carries a $151 \mathrm{~kb}$ de novo deletion on chromosome 13 q34 including 3 genes. None of these genes has been previously implicated in neuropsychiatric disorders.

Rare inherited copy number variants in ASD candidate genes. In addition to the CNVs overlapping known genomic disorders, we observed 24 inherited CNVs from 23 probands, intersecting ASD candidate genes. The clinical features of ASD probands and the details of the CNVs overlapping SFARI genes are reported in the Supplementary Table S5.

Among these CNVs, a notable case finding is the identification of an intragenic multiexonic deletion of the VPS13B gene, classified as a syndromic ASD candidate gene in the SFARI database, in proband AB151 (Fig. 2). By real time qPCR we confirmed that the deletion is inherited from the unaffected mother and includes exons 23-35 


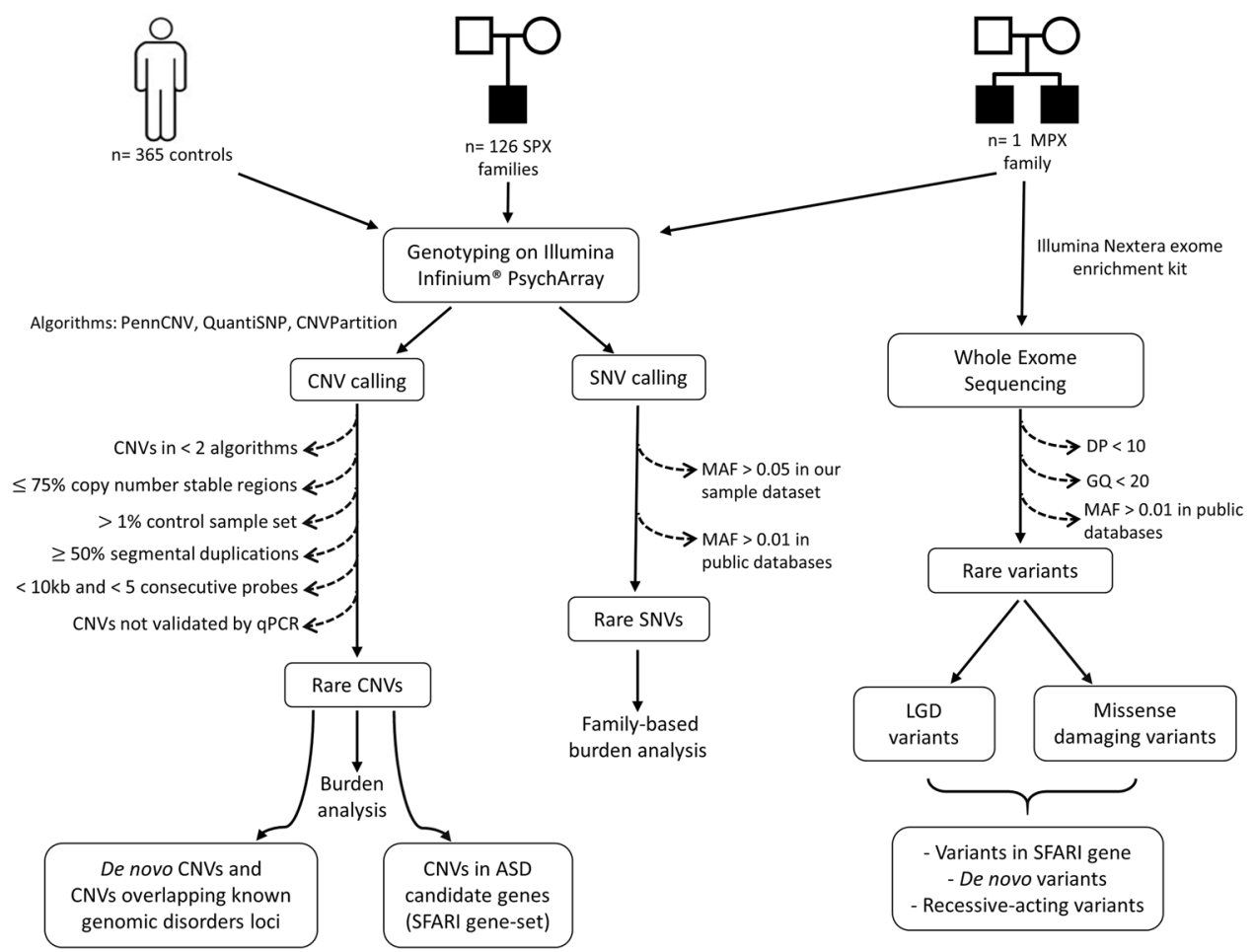

Figure 1. Schematic of the experimental design.

\begin{tabular}{|l|l|l|l|l|l|l|l|}
\hline & $\begin{array}{l}\text { ASD cases } \\
(\mathbf{n}=\mathbf{1 2 8})\end{array}$ & $\begin{array}{l}\text { Controls } \\
(\mathbf{n}=\mathbf{3 6 3})\end{array}$ & $\mathbf{P}$ value & & $\begin{array}{l}\text { ASD cases } \\
(\mathbf{n}=\mathbf{1 2 8})\end{array}$ & $\begin{array}{l}\text { Controls } \\
(\mathbf{n}=363)\end{array}$ & P value \\
\hline All CNVs & & & & Deletions & & & \\
\hline$N$ & 253 & 639 & & N & 134 & 295 & \\
\hline RATE & 1.977 & 1.76 & 0.073793 & RATE & 1.047 & 0.8127 & $\mathbf{0 . 0 0 9 3 9 9}$ \\
\hline PROP & 0.8984 & 0.8154 & $\mathbf{0 . 0 1 9 0 9 8}$ & PROP & 0.6641 & 0.5372 & $\mathbf{0 . 0 0 8 0 9 9}$ \\
\hline TOTKB & 296.1 & 352.6 & 0.823018 & TOTKB & 166.4 & 226.2 & 0.815718 \\
\hline AVGKB & 143.5 & 156.3 & 0.679432 & AVGKB & 112.9 & 144 & 0.720728 \\
\hline SFARI genes, all CNVs & & & & SFARI genes, deletions & & & \\
\hline N & 33 & 57 & & N & 23 & 36 & \\
\hline GRATE & 0.2578 & 0.157 & $\mathbf{0 . 0 3 9 8 9 6}$ & GRATE & 0.1797 & 0.09917 & $\mathbf{0 . 0 4 2 1 9 6}$ \\
\hline GPROP & 0.1953 & 0.1295 & 0.050795 & GPROP & 0.08264 & $\mathbf{0 . 0 1 5 1 9 9}$ \\
\hline
\end{tabular}

Table 2. Rare CNV Burden Analysis in Cases and Controls. N: Number of events; RATE: Number of CNVs per person; PROP: Proportion of cases/controls to have at least one CNV; TOTKB: Total kb length spanned per person; AVGKB: Average segment size per person; GRATE: Number of SFARI genes spanned by CNVs per person; GPROP: Proportion of cases/controls to have CNVs spanning at least one SFARI gene.

of the VPS13B full-length isoforms (1-62 exons, NM_152564.4 and NM_017890.4). To assess if the identified deletion affects VPS13B expression, we performed a qPCR on cDNA samples of proband AB151 and his mother, and two controls. A significant reduced expression of VPS13B full-length isoforms was observed in the mother and in the proband, confirming that the deletion causes a decrease of VPS13B full-lenghth isoforms. The proband has ASD with an impaired adaptive functioning, and underwent surgery for trigonocephaly at age 4 months.

A second notable CNV is a CNTNAP2 deletion in proband AB87, a well-established ASD candidate gene (SFARI score $2 \mathrm{~S})^{22}$ (Fig. 3).

Furthermore, we identified $8 \mathrm{CNV}$ s overlapping ASD genes with a suggestive evidence supporting their link to ASD (SFARI score $=3$ ) and $14 \mathrm{CNVs}$ intersecting genes implicated in ASD with minimal evidence (SFARI score $=4$ ). The most notable CNVs in these groups included (Fig. 3):

(a) Two inherited deletions overlapping exon 2 and/or exon3 of the PARK2 gene have been identified in cases $\mathrm{AB} 47$ and $\mathrm{AB} 156$.

(b) Two different maternally inherited deletions in RBFOX1 have been identified in two ASD subjects: one in case AB74 including RBFOX1 intron 2, the other one in proband AB86 encompassing the noncoding exon 1 of RBFOX1 transcript variant 6 (NM_001142334.1). 


\begin{tabular}{|c|c|c|c|c|c|c|c|c|c|c|}
\hline Cytoband & Coordinates $\min / \max$ & $\begin{array}{l}\text { length (bp) } \\
\mathrm{min} / \max \end{array}$ & $\mathrm{CN}$ & Sample Sex & Inheritance & Genes & Freq $^{a}$ & Penetrance $\%^{11}$ & $\begin{array}{l}\text { Clinical } \\
\text { Diagnosis }\end{array}$ & $\begin{array}{l}\text { Other rare genic } \\
\text { CNVs }\end{array}$ \\
\hline \multicolumn{11}{|c|}{ a) CNVs Overlapping with Known Genomic Disorders } \\
\hline $1 \mathrm{p} 36.32$ & chr1:2473258-3118326 & 645069 & 1 & AB27 Male & De novo & 9 genes & 0 & $\begin{array}{l}\text { 1p36 del (100\% penetrance in } \\
\text { DD/ASD/CM) }\end{array}$ & PDD & \\
\hline $15 q 11.2$ & $\begin{array}{l}\text { chr15:22755185-23228712/ } \\
\text { chr15:22383300-23668092 }\end{array}$ & $\begin{array}{l}473528 / \\
1284793\end{array}$ & 3 & AB111 Male & Maternal & 10 genes & $4(1 \mathrm{TP}, 2 \mathrm{NP}, 1 \mathrm{C})$ & $\begin{array}{l}15 q 11.2 \text { dup (1.8\% penetrance } \\
\text { for ASD })^{17}\end{array}$ & PDD & \\
\hline 16 p13.11 & $\begin{array}{l}\text { chr16:15493046-16301530/ } \\
\text { chr16:15395596-16859425 }\end{array}$ & $\begin{array}{l}808485 / \\
1463830\end{array}$ & 3 & AB35 Female & Paternal & 18 genes & $2(1 \mathrm{TP}, 1 \mathrm{C})$ & $\begin{array}{l}\text { 16p13.11 dup (8.4\% } \\
\text { penetrance in DD/ASD/CM) }\end{array}$ & PDD & $\begin{array}{l}\text { (chr10:68065751- } \\
\text { 68180999)xlmat }\end{array}$ \\
\hline $17 \mathrm{q} 12$ & $\begin{array}{l}\text { chr17:34815551-36249430/ } \\
\text { chr17:34461869-36455348 }\end{array}$ & $\begin{array}{l}1433880 / \\
1993480\end{array}$ & 3 & AB164 Male & Maternal & 11 genes & $1(\mathrm{TP})$ & $\begin{array}{l}\text { 17q12 dup (17\% penetrance in } \\
\text { DD/ASD/CM) }\end{array}$ & PDD & $\begin{array}{l}\text { (chr11:4387760- } \\
\text { 4409718)x3mat }\end{array}$ \\
\hline $22 q 13.33$ & $\begin{array}{l}\text { chr22:50814075-51181759/ } \\
\text { chr22:50764057-51304566 }\end{array}$ & \begin{tabular}{|l}
$367684 /$ \\
540509
\end{tabular} & 1 & AB133 Female & De novo & 19 genes & 0 & $\begin{array}{l}\text { Phelan-McDermid del }(100 \% \\
\text { penetrance in DD/ASD/CM) }\end{array}$ & ASD & $\begin{array}{l}\text { (chrX:38490844- } \\
\text { 38624791)x3mat }\end{array}$ \\
\hline \multicolumn{11}{|c|}{ b) Rare De Novo genic CNVs } \\
\hline $5 \mathrm{p} 13.2$ & chr5:35730729-35991299 & 260571 & 3 & AB161 Male & De novo & $\begin{array}{l}\text { CAPSL, IL7R, } \\
\text { SPEF2, UGT3A1 }\end{array}$ & 0 & & PDD & $\begin{array}{l}\text { (chr16:3010466- } \\
\text { 3032566)x1mat; } \\
\text { (chr1:236850052- } \\
\text { 237167218)x3pat }\end{array}$ \\
\hline $13 q 34$ & $\begin{array}{l}\text { chr13:114323997- } \\
114475037\end{array}$ & 151041 & 1 & AB84 Male & De novo & $\begin{array}{l}\text { FAM70B, GRK1, } \\
\text { LOC100130386 }\end{array}$ & 0 & & PDD & \\
\hline
\end{tabular}

Table 3. CNVs overlapping with known genomic disorders loci (a) and de novo CNVs (b). ${ }^{a}$ Frequency a in 363 controls (C) and 238 parents (P): TP: transmitting parent; NP: non-transmitting parent. ${ }^{\text {bPDD: pervasive }}$ developmental disorder according to the DSM-IV; ASD: autism spectrum disorder according to the DSM-5.

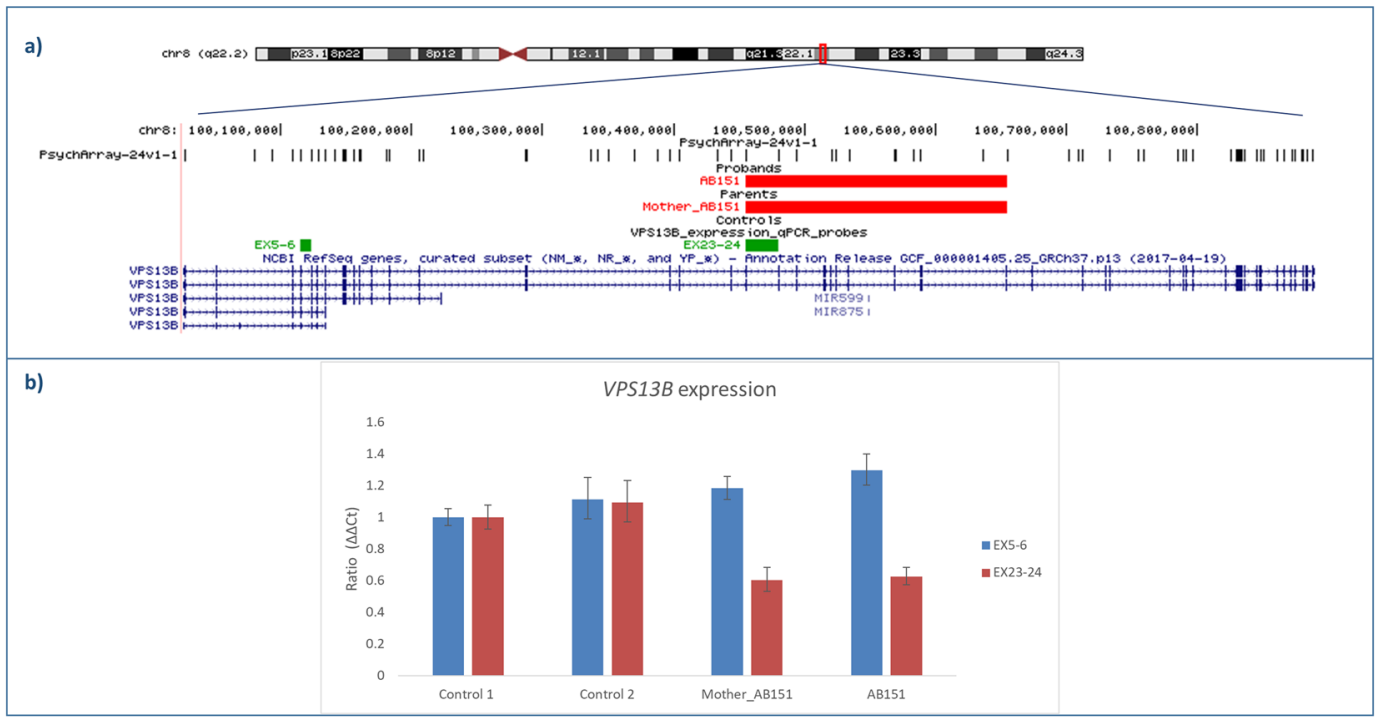

Figure 2. VPS13B deletion. (a) UCSC hg19 screenshot showing the $200 \mathrm{~kb}$ maternally inherited deletion impacting the VPS13B gene identified in case AB151. No CNVs in VPS13B have been detected in our control cohort. qPCR probes used to test VPS13B expression are shown in green; (b) VPS13B expression levels in the deletion carriers (AB151 and the mother of $\mathrm{AB} 151)$ and in two controls.

(c) One maternally inherited deletion involving the last exon of the two shorter gene transcript variants of the WWOX gene (NM_130791.3 and NR_120436.1) has been identified in proband AB139.

(d) A paternally inherited deletion involving MACROD2 exon 6 (NM_080676.5) has been identified in case AB81.

(e) A large paternally inherited deletion, located on the proximal region of the long arm of chromosome 15, was identified in case AB63. The minimal deleted region encompasses nine genes, including the two ASD candidate genes, NDNL2 and APBA2 (SFARI score =4).

(f) Two maternally inherited deletions encompassing intron 11 and exon 12 of the CTNNA3 gene have been identified in cases AB119 and AB35, respectively. It is worthy of note that case AB35 also carries a large paternally inherited duplication encompassing the region $16 \mathrm{p} 13.11$, associated with a wide range of neurodevelopmental disorders (Table 3a). Since we have previously implicated CTNNA3 as a candidate gene in ASD acting in a recessive mode of inheritance ${ }^{23}$, we also investigated if the exonic deletion in case AB35 could act by unmasking rare variants in the non-deleted allele. However, sequence analysis of the entire CTNNA3 coding sequence in case $\mathrm{AB} 35$ and in his parents, did not identify rare (MAF $<1 \%$ ) exonic variants. We identified seven CTNNA3 deletions in 6 controls and in one non-transmitting mother, consistent with the previously reported evidence that heterozygous deletions in CTNNA3 are not pathological ${ }^{23}$. 


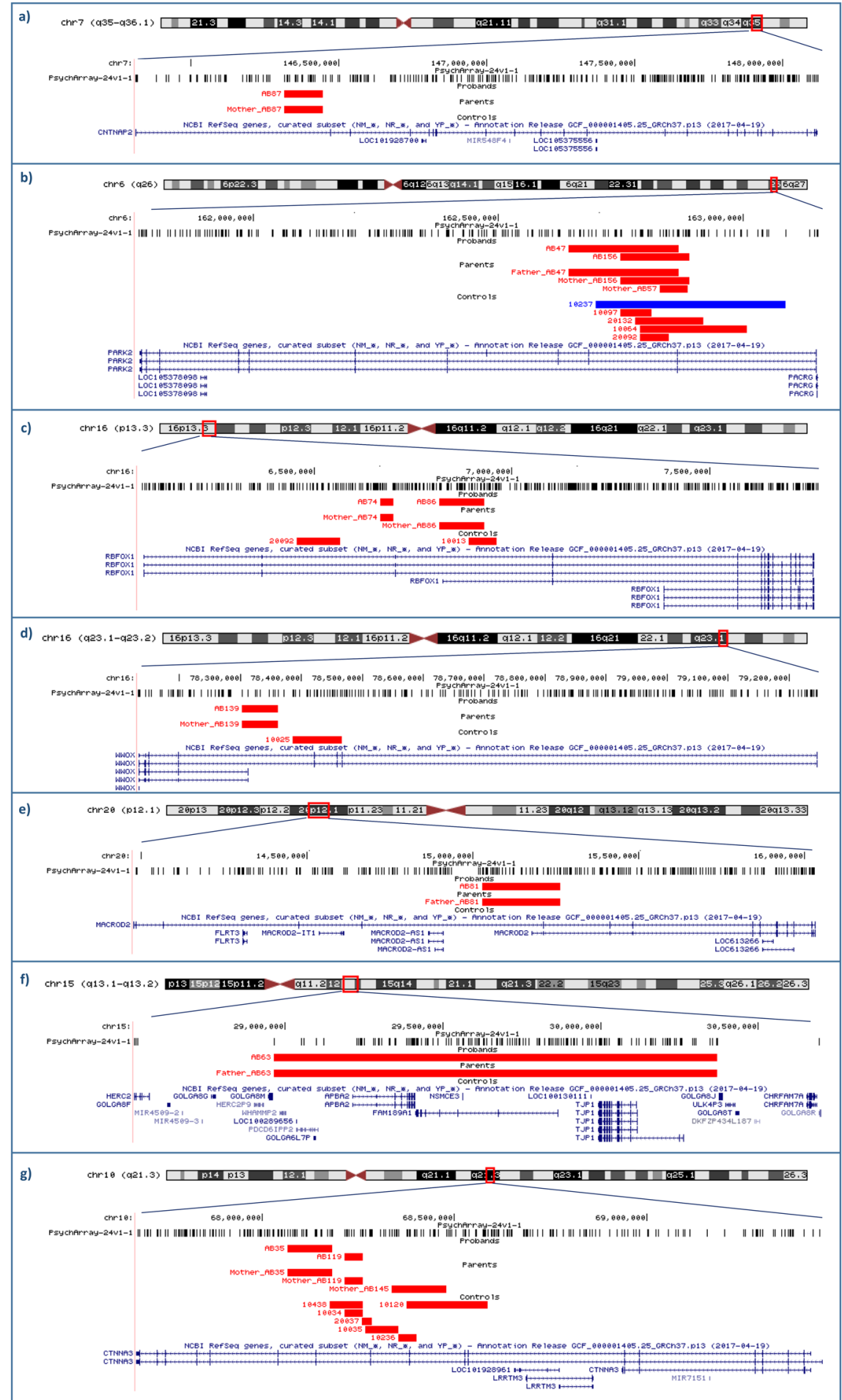

Figure 3. Most notable CNVs intersecting SFARI genes. UCSC hg19 screenshots reporting the most notable CNVs impacting SFARI genes identified in our ASD sample and in controls. PsychArray probes are shown. (a) A $128 \mathrm{~kb}$ maternally inherited intronic deletion in the CNTNAP2 gene in case AB87. No CNVs in CNTNAP2 have been detected in our control cohort; (b) PARK2 CNVs in 2 cases (AB47 and AB156) and 5 controls; (c) RBFOX1 deletions in 2 cases (AB74 and AB86) and 2 controls; (d) WWOX non overlapping deletions in case $\mathrm{AB} 139$ and in one control subject; (e) A $236 \mathrm{~kb}$ paternally inherited deletion in the MACROD2 gene in case AB81. No CNVs in MACROD2 have been detected in our control cohort; (f) A 1.4-2.2 Mb paternally inherited deletion mapping in 15q13.1-q13.2 locus and impacting at least nine genes, including NDNL2 and APBA2. No CNVs in this locus have been found in our control sample; (g) CTNNA3 deletions in 2 cases (AB35 and AB119), one non-transmitting mother (mother of case AB145) and 6 controls. 
Rare genic SNV analysis. Leveraging the presence of probes for over 250,000 for putative functional exonic variants on the Psych-array, as well as 50,000 markers selected based on evidence from prior genetic studies of psychiatric disorders, we used genotyping data to perform an association analysis focused on rare genic variants.

Since single variant analysis was unfeasible due to lack of power, we carried out a global burden analysis. In order to avoid the problem of cryptic stratification between cases and controls, that is particularly relevant in rare variants analysis because the spectrum of rare variation can differ greatly between populations, we adopted a more robust family-based approach. After pruning for variants in linkage disequilibrium (LD), we tested the total burden of 51,547 genic rare variants using an extension of the transmission disequilibrium test (TDT-BRV) ${ }^{24}$, that revealed a significant excess transmission for the rare alleles from heterozygous parents to affected offspring $(129,177$ transmitted versus 127,906 untransmitted rare alleles, TDT $\mathrm{p}=0.0122)$.

Exome sequencing. In the single multiplex family included in our study (AB162/AB163), CNV analysis did not detect any potentially relevant $\mathrm{CNV}$. Thus, exome sequencing was performed to test if point mutations might explain the ASD phenotype shared by the two affected brothers. We prioritized 39 exonic rare variants predicted deleterious and belonging to one of three "high-risk" categories: (i) SFARI gene-set; (ii) recessive-acting variants; (iii) de novo variants (Supplementary Table S6). We observed that about $56 \%$ (22 out of 39) of the prioritized rare variants have been also genotyped in the PsychArray. This provided useful information about the frequency of these variants in the entire sample of cases and controls drawn from the same population.

We identified 30 Likely Gene Disrupting (LGD) or damaging missense variants in ASD candidate genes, among which 10 are shared by both affected siblings. We highlight a shared damaging missense mutation, affecting GPHN, a neuronal synaptic gene predicted to be haploinsufficient $(\mathrm{pLi} \text { score }=0.999981)^{25}$.

Moreover, we identified four X chromosome hemizygous damaging missense variants affecting the FAAH2, RNF113A, SHROOM2, MID1IP1 genes, shared by the two affected brothers. Both nonsynonymous variants in RNF113A and MID1IP1 were identified in another ASD family and were never found in our control cohort. The FAAH2 missense variant was more frequent in cases versus controls (Fisher $P=0.00085$ ). Thus, these $\mathrm{X}$-linked variants could contribute to ASD susceptibility.

Finally, we discovered a de novo frameshift variant in the TRPV1 gene, present in only one of the affects sibs (AB162). This $1 \mathrm{bp}$-deletion in the TRPV1 gene causes the introduction of 2 novel amino acids followed by a premature termination codon, probably inducing nonsense-mediated-decay.

\section{Discussion}

Considerable advances have been made, over the past two decades, in understanding the genetic architecture of ASD. While a substantial proportion of ASD heritability is explained by common polygenic variation, rare variants have been shown to account for a considerable proportion of ASD cases, thus offering distinctive opportunities to understand ASD-related biology. In particular, the number of genes found to confer ASD liability has dramatically increased thanks to the identification of highly penetrant rare variants through high-resolution microarrays and exome/genome sequencing studies.

The main aim of this study was to analyze rare genetic variants in a homogeneous cohort of ASD families of Italian origin, in order to assess their contribution to ASD risk. Specifically, we focused on CNVs as they exert a more direct gene dosage impact and have been generally implicated in psychiatric diseases with larger effect size compared to SNPs. Moreover, we tested a set of exonic rare single nucleotide variants included in the Illumina PsychArray, enriched for variants previously implicated in neuropsychiatric disorders.

It is well-known that $\mathrm{CNV}$ detection using SNP-arrays varies widely in number of $\mathrm{CNV}$ calls, $\mathrm{CNV}$ size range, percentage of non-validated CNVs, and CNV type (exonic vs non-genic CNVs) across different array platforms. Therefore, the advantages and the limitations of each array should carefully be weighted, for an appropriate array selection. We chose the Illumina PsychArray for several reasons. First, even if this array has a lower genome-wide coverage in comparison to other high-density arrays, we demonstrate that the specificity of the detection of genic $\mathrm{CNV}$ using this array is very high. According to our experimental validation results, PsychArray data analyzed with our CNV calling algorithm and fulfilling criteria for stringent CNV calls, led to specific detection of genic CNVs down to $10 \mathrm{~kb}$. Second, the Illumina PsychArray is enriched for exome variants and particularly protein altering variants implicated in psychiatric disorders and segregating at low allele frequencies in the genome. Therefore, this array offers an economical method to test the aggregate effect of rare variants in specific genes or gene sets. Obviously, a disadvantage of the PsychArray is the limited coverage of intergenic regions; however, we prioritized genic CNVs as they are generally more directly linked to pathogenic effects, while the interpretation of CNVs in non-genic regions still remains a challenging task. Another potential limitation of the PsychArray is its lower sensitivity compared to arrays with higher SNP coverage, as reported in a previous study that compared the performance of different arrays for genome-wide CNV detection ${ }^{11}$. However, the low sensitivity is likely to be confined to the detection of non-genic CNVs, given the reduced SNP coverage in intergenic regions. Moreover, in our study, a combination of multiple CNV detection algorithms was applied in order to obtain sensitive and reliable CNV calls, while the above-mentioned study applied only one analysis software at a time ${ }^{11}$. In order to test the PsychArray sensitivity in genic regions, we compared CNV calls detected by the PsychArray with calls from a high-resolution array in an ASD sample genotyped by both arrays. The PsychArray allowed the identification of all rare genic CNVs, including a clinically relevant CHRNA7 duplication. Therefore, the false negative rate of genic CNV detection is not likely to represent a relevant issue for the aim of this study; in particular, given the PsychArray enrichment for probes in genes previously involved in psychiatric disorders, CNVs spanning ASD relevant genes are expected to be identified with a higher sensitivity.

However, the uneven distribution of SNPs and the requirement of an accurate multi-algorithms analysis currently prevent the application of the PsychArray in clinical practice, where the use of more robust and standardized microarrays is needed. 
Our genome-wide CNV analysis identified 5 large clinically relevant CNVs, overlapping loci previously implicated in known genomic disorders. Among them, the two identified deletions are de novo and therefore more likely to be pathogenic ${ }^{7}$. Instead, the three recurrent large duplications exhibit reduced penetrance, as they are inherited from a phenotypically normal parent and, in two cases, they were also identified in our control sample (Table 3a). Consistent with an oligogenic CNV model, it is likely that these primary recurrent CNVs interact with other etiologic risk variants at other loci to exert phenotypic effect. Variants in the genetic background can indeed modulate the effects of recurrent CNVs, ultimately defining the phenotypic trajectory in CNV carriers, as it has been recently demonstrated for $16 \mathrm{p} 11.2$ deletion carriers ${ }^{26}$.

We also exploited CNV data to assess the aggregate effect of CNVs in cases and controls. Consistent with previous studies $5,27,28$, we found an increased global rare CNV burden in cases compared to controls, more notably for deletions. Furthermore, we identified a significant enrichment of rare CNVs intersecting ASD candidate genes. Notably, further support to the role of rare variants in ASD susceptibility came also from the family-based analysis of genic rare SNVs genotyped by the Illumina PsychArray.

Next, we took into consideration individual CNVs identified in our cohort, in order to investigate their potential relevance to the ASD phenotype based on gene constrain measures, CNV frequency in cases and controls, $\mathrm{CNV}$ location in the gene and relevant literature. $9 \mathrm{CNV}$ s overlapping genes VPS13B, WWOX, CNTNAP2, RBFOX1, MACROD2, APBA2, and PARK2 were of particular interest in our cohort (Supplementary Table S5).

Mutations in VPS13B have been associated with Cohen syndrome, a rare autosomal recessive neurodevelopmental disorder, and recessive variants have been reported in cases of ASD or ID with autistic features ${ }^{29,30}$. We showed that the inherited deletion identified in case AB151 causes a significant reduced expression of VPS13B full-length isoforms implicated in Cohen syndrome ${ }^{31}$, supporting the hypothesis that genes known to cause severe syndromes when completely knocked-out, might determine milder ASD phenotypes when only partially inactivated $^{29}$.

Consistent with the above model, we identified an intragenic deletion of WWOX in case AB139, with a high-functioning ASD. Biallelic mutations in WWOX are responsible for early infantile epileptic encephalopathy-28 (EIEE28; OMIM 616211) and autosomal recessive spinocerebellar ataxia-12 (SCAR12; OMIM 614322). Rare CNVs overlapping WWOX have been reported at greater frequency in ASD cases versus unaffected controls ${ }^{6}$, thus suggesting that $W W O X$ heterozygous variants act as weak risk factors, generally associated with milder ASD phenotypes, as in our case.

A third syndromic gene affected by a deletion in our sample is CNTNAP2. Homozygous or compound heterozygous mutations in CNTNAP2 are the cause of cortical dysplasia-focal epilepsy syndrome (CDFES; OMIM 610042 ) and this gene has been implicated in multiple neurodevelopmental disorders, including autism and ID ${ }^{22}$. The inherited deletion identified in proband AB87 maps in CNTNAP2 intron 1, without affecting the previously described binding site for the transcription factor FOXP2 that should regulate its expression ${ }^{32}$. A recent comprehensive cross-disorder analysis of CNTNAP2 role in psychiatric disorders ${ }^{33}$ suggested that CNTNAP2 is unlikely to be a primary risk gene for psychiatric disorders, however this CNV might contribute to ASD risk interacting with other rare or common variants.

RBFOX1 regulates alternative splicing events of genes critical for neuronal development and it has been strongly implicated in the etiopathogenesis of a wide spectrum of neurodevelopmental disorders including ASD. In particular, RBFOX1 CNVs have been highlighted in individuals with ASD and other neuropsychiatric disorders; ${ }^{34,35}$ transcriptomic analyses of autistic postmortem brains revealed a reduced expression of RBFOX 1 in ASD patients which possibly results in altered splicing of RBFOX1 target exons in synaptic genes ${ }^{36}$. In our study we identified two maternally inherited RBFOX1 deletions in cases and two in controls. A similar RBFOX1 deletion was reported in an autism family by whole genome sequencing in combination with a rare NTM deletion inherited from the other parent ${ }^{37}$. Interestingly, our proband AB86 also carries a paternal duplication in the ASD candidate gene SNTG2, supporting an oligogenic model for ASD risk in this family.

MACROD2 gene has been implicated in ASD susceptibility by both CNVs and GWAS studies ${ }^{3,38,39}$. Moreover, a SNP in MACROD2 was significantly associated with autistic-like traits in general population ${ }^{40}$. Therefore, the exonic MACROD2 deletion in case AB81 is of particular interest, especially given the absence of MACROD2 CNVs in our control sample, as well as in the CNV map of the human genome ${ }^{41}$.

CNVs at 15q13.1-q13.2 locus are likely due to non-allelic homologous recombination (NAHR) events between segmental duplications in proximal chromosome $15 \mathrm{q}$ breakpoints 3 and 4 (BP3-BP4 CNVs). Unlike the more common clinically relevant BP1-BP3 CNVs (PWS/AS critical region) and BP4-BP5 CNVs (15q13.3 microdeletion syndrome), BP3-BP4 CNVs have unclear significance given their rarity and the imperfect segregation with disease status in families with ID and/or developmental delay ${ }^{42,43}$. However, the presence of common clinical features among cases with BP3-BP4 deletions and the presence of genes with roles in development and nervous system function in the deletion region, suggest that this deletion may have a role in abnormal phenotypes in some individuals ${ }^{44}$. The most notable gene included in case AB63 deletion is APBA2, encoding a neuronal adapter protein essential for synaptic transmission ${ }^{45}$. Of note, our patient had hypotonia particularly involving the facial muscles.

CNVs in PARK2 have been described in individuals with ASD and other neurodevelopmental disorders ${ }^{46-48}$ suggesting their pathogenic role in multiple brain processes. However, PARK2 CNVs have also been found in control populations and an accurate analysis of the CNV location showed that, while CNVs targeting PARK2 exons 5-12 are significantly more frequent in neurodevelopmental disorders cases than in controls, CNVs involving exons 2-4 are well-tolerated and very common both in cases and controls ${ }^{48,49}$. All PARK2 deletions identified in our study map between exons 2 and 3 and their frequency is the same in cases and controls, suggesting that they do not represent major risk factors for ASD in these two ASD families.

The multiplex family included in our study (AB162/AB163) was further investigated by exome sequencing. The most interesting WES finding is an inherited missense variant in the GPHN gene, which is shared by both 
affected siblings. GPHN is a haploinsufficient gene encoding a key scaffolding protein in the neuronal postsynaptic membrane, with well-established functional links with synaptic proteins implicated in ASD, such as neuroligins and neurexins ${ }^{50}$. Moreover, GPHN deletions have been identified in ASD subjects and have been reported to be associated to a diverse range of neurodevelopmental conditions ${ }^{50,51}$. Among the chromosome X hemizygous variants shared by both affected brothers, we highlighted a missense variant in the FMRP-target gene RNF113A ${ }^{21}$. Interestingly, a RNF113A stop-gain mutation has been associated with an X-linked form of trichothiodystrophy (TTD), a disease characterized by a wide range of clinical features, including intellectual and developmental disabilities $^{52}$. Finally, we identified a de novo frameshift variant in the TRPV1 gene, only present in case AB162. $T R P V 1$ is a non-selective cation channel highly expressed in the brain, where it participates in several synaptic functions, such as modulation of spine morphology, synaptic transmission and plasticity ${ }^{53}$. TRPV1 plays an important role in the transmission and modulation of pain and it might be involved in the altered pain sensitivity often observed in SHANK3-related ASD, due to SHANK3 role in the modulation of TRPV1 function and pain transduction ${ }^{54}$. Therefore, we investigated if self-injurious behaviours had been reported for case AB162, and/ or if the 2 ASD siblings, discordant for the presence of the disruptive TRPV1 mutation, could have a different pain tolerance threshold. However, no evidence of altered behavioural responses to pain (tested according to the standard neurological examination) was observed in case AB162, suggesting that TRPV1 is haplosufficient for the pain-sensitivity phenotype. However, given the involvement of TRPV1 multiple functions in the nervous system, it is still possible that the de novo TRPV1 frameshift variant could contribute to modulation of the ASD phenotype.

In conclusion, this study adds relevant data to the large number of CNV studies in autism, all of which are critical for accurate interpretation of results, given the complex contribution of CNVs to ASD etiology. We have also shown that the Illumina PsychArray is a reliable and cost-efficient tool for genic CNVs detection, in addition to providing useful genotyping data, including rare and low-frequency exonic variants previously implicated in psychiatric disorders.

A major limitation in our study is the relatively small sample size, which provides limited power to confidently identify novel rare risk variants for ASD. Therefore, to prioritize variants of interest we relied on previously identified ASD candidate genes. Our data support the contribution of VPS13B, WWOX, CNTNAP2, RBFOX1, MACROD2, APBA2, PARK2, GPHN, and RNF113A in ASD susceptibility. Moreover, our study was successful in providing evidence for an enrichment of rare CNVs intersecting SFARI genes and a global increased burden of rare variants in ASD cases compared to controls, thus providing further support for a multigenic model of ASD risk.

\section{Methods \\ Study design. An overview of the experimental design is shown in Fig. 1. Briefly, all DNA samples from 128 ASD individuals, 238 parents and 365 controls were genotyped using the Illumina Infinium ${ }^{\circledR}$ PsychArray microarrays (Illumina, San Diego, California, USA). After quality controls, genotyping data were used to assess the combined contribution of rare CNVs and rare exome variants in this cohort of ASD families. Moreover, we undertook exome sequencing (WES) in the single multiplex family included in this study to test if rare coding mutations might explain the ASD phenotype shared by the two affected brothers in this family.}

Description of sample. A total of 128 Italian individuals with an ASD diagnosis and 238 parents from 127 unique families were recruited at the IRCCS Stella Maris Foundation (Pisa, Italy). All participants were of self-reported Italian ancestry and provided a written informed consent to participate. This sample is independent of the Italian sample included in the Autism Genome Project study ${ }^{5,27}$. See Supplementary Methods for clinical assessment details.

The ASD samples include 106 males and 22 females, with a 4.8:1 male/female ratio. DNA samples from both proband's parents were available for 111 families, and from a single parent for the remaining ones. All DNA samples were extracted from whole blood.

The main clinical and diagnostic characteristics of all ASD probands are summarized in Table 1.

The control sample consisted of 365 anonymized DNA samples from Italian individuals (M/F:199/166), with no psychiatric disorders (See Supplementary Methods).

Genotyping and CNV data analysis. All DNA samples from 128 ASD individuals, 238 parents and 365 controls were genotyped using the Illumina Infinium ${ }^{\circledR}$ PsychArray microarrays (Illumina, San Diego, California, USA) in two batches, the first using Illumina PsychArray-24 v1.0, the second using PsychArray-24 v1-1 array.

Genotyping, CNV calling methods and quality control (QC) criteria at both sample-level and CNV call-level are described in Supplementary Methods.

After QC, 729 samples (128 ASD individuals, 238 parents and 363 controls) remained.

Burden analyses for rare CNVs in cases and controls were performed using PLINK v1.07 ${ }^{55}$

To determine whether the observed CNV enrichment is really specific to the subset of ASD candidate genes and not general to all genes, we applied the gene-set enrichment method for CNV data ${ }^{56}$ implemented in the PLINK software package (-cnv-enrichment-test), that is robust to case-control differences in CNV size, CNV rate, and systematic differences in gene size. Given the relatively small sample size, we applied robust permutation testing (-mperm 10000) and 1-sided empirical p-values were returned.

As CNV burden analysis is highly susceptible to technical bias, to ensure that CNV ascertainment was consistent among affected individuals and control subjects for which we do not have parental data, we included in the analysis only the CNVs identified in cases before running the "trio option" in PennCNV. 
Burden analysis of single-nucleotide rare variants. Prior to association analysis, additional quality control was performed using PLINK v.1.9. ${ }^{57}$. Since the genotyping was carried out in in two batches, we limited our analysis to the 566,178 variants present in both versions of the array. Moreover, in order to deal with differences in SNP names and reference allele, we took advantage of the Illumina PsychArray support files and then we specifically checked: i) SNP allele frequency differences between the two batches, ii) the presence of SNPs with different names but mapping to the same genomic position, iii) SNP strand differences between the two batches using the-flip-scan option. Then, SNPs were filtered on missing rate (genotyping missingness $>5 \%$ ), missing rate differences between cases and controls $(p<0.001)$, and deviations from Hardy-Weinberg equilibrium $(p<0.001)$. Post QC, non-monomorphic SNPs were mapped to RefSeq genes and association analysis was performed on rare genic variants. For frequency filtering we retained only variants with a MAF $\leq 0.05$ in our dataset of 748 samples (128 ASD individuals, 238 parents and 382 controls) and a MAF $\leq 0.01$ in gnomeAD exome, gnomeAD genome (https://gnomad.broadinstitute.org/) and the 1000 Genomes Project (http://www.internationalgenome.org/).

Finally, we generated a final subset of SNPs pruned for linkage disequilibrium ( $200 \mathrm{~kb}$ window size, $\left.\mathrm{r}^{2}>0.5\right)$, in order to avoid inflation of type I error due to presence of intermarker LD.

Rare variant global burden association analysis was performed using the Burden of Rare Variants Transmission Disequilibrium Test (TDT-BRV), an extension of the TDT. The TDT-BRV method counts the number of minor-allele-transmitted events and major-allele-transmitted events from every informative parent to the affected proband ${ }^{24}$.

Ethical approval and informed consent. All participants provided a written informed consent to participate to this study. This study was approved by the local Ethical Committee (Fondazione Stella Maris, IRCCS; protocol number 05/2011). All research was performed in accordance with the relevant guidelines and regulations.

\section{Data availability}

All data generated or analysed during this study are included in this published article and its Supplementary Information files.

Received: 5 September 2019; Accepted: 19 January 2020;

Published online: 21 February 2020

\section{References}

1. Lyall, K. et al. The Changing Epidemiology of Autism Spectrum Disorders. Annu Rev Public Health 38, 81-102 (2017).

2. Gaugler, T. et al. Most genetic risk for autism resides with common variation. Nat Genet 46, 881-885 (2014).

3. Grove, J. et al. Identification of common genetic risk variants for autism spectrum disorder. Nat Genet 51, 431-444 (2019).

4. Krumm, N. et al. Transmission disequilibrium of small CNVs in simplex autism. Am J Hum Genet 93, 595-606 (2013).

5. Pinto, D. et al. Convergence of Genes and Cellular Pathways Dysregulated in Autism Spectrum Disorders. Am J Hum Genet, (2014).

6. Leppa, V. M. et al. Rare Inherited and De Novo CNVs Reveal Complex Contributions to ASD Risk in Multiplex Families. Am J Hum Genet 99, 540-554 (2016).

7. Miller, D. T. et al. Consensus statement: chromosomal microarray is a first-tier clinical diagnostic test for individuals with developmental disabilities or congenital anomalies. Am J Hum Genet 86, 749-764 (2010).

8. Battaglia, A. et al. Confirmation of chromosomal microarray as a first-tier clinical diagnostic test for individuals with developmental delay, intellectual disability, autism spectrum disorders and dysmorphic features. Eur J Paediatr Neurol 17, 589-599 (2013).

9. Volkmar, F. et al. Practice parameter for the assessment and treatment of children and adolescents with autism spectrum disorder. $J$ Am Acad Child Adolesc Psychiatry 53, 237-257 (2014).

10. Stamouli, S. et al. Copy Number Variation Analysis of 100 Twin Pairs Enriched for Neurodevelopmental Disorders. Twin Res Hum Genet 21, 1-11 (2018).

11. Haraksingh, R. R., Abyzov, A. \& Urban, A. E. Comprehensive performance comparison of high-resolution array platforms for genome-wide Copy Number Variation (CNV) analysis in humans. BMC Genomics 18, 321 (2017).

12. Rodriguez-Lopez, J. et al. Genome wide analysis of rare copy number variations in alcohol abuse or dependence. J Psychiatr Res $\mathbf{1 0 3}$, 212-218 (2018).

13. Kirov, G. et al. The penetrance of copy number variations for schizophrenia and developmental delay. Biol Psychiatry 75, 378-385 (2014).

14. Tabet, A. C. et al. A framework to identify contributing genes in patients with Phelan-McDermid syndrome. NPJ Genom Med 2, 32 (2017).

15. Battaglia, A. et al. Further delineation of deletion 1 p36 syndrome in 60 patients: a recognizable phenotype and common cause of developmental delay and mental retardation. Pediatrics 121, 404-410 (2008).

16. Battaglia, A. in GeneReviews(R), Adam, M. P. et al. Eds. (University of Washington, SeattleUniversity of Washington, Seattle. GeneReviews is a registered trademark of the University of Washington, Seattle. All rights reserved., Seattle (WA), 2008 [updated 2013]).

17. Mitchell, E. et al. Recurrent duplications of 17q12 associated with variable phenotypes. Am J Med Genet A 167a, 3038-3045 (2015).

18. Tropeano, M. et al. Male-biased autosomal effect of 16p13.11 copy number variation in neurodevelopmental disorders. PLoS One 8, e61365 (2013).

19. Chaste, P. et al. Modest impact on risk for autism spectrum disorder of rare copy number variants at $15 \mathrm{q} 11.2$, specifically breakpoints 1 to 2. Autism Res 7, 355-362 (2014).

20. Sironen, A. et al. Loss of SPEF2 function in mice results in spermatogenesis defects and primary ciliary dyskinesia. Biol Reprod $\mathbf{8 5}$, 690-701 (2011).

21. Ascano, M. Jr. et al. FMRP targets distinct mRNA sequence elements to regulate protein expression. Nature 492, 382-386 (2012).

22. Penagarikano, O. \& Geschwind, D. H. What does CNTNAP2 reveal about autism spectrum disorder? Trends Mol Med 18, 156-163 (2012).

23. Bacchelli, E. et al. A CTNNA3 compound heterozygous deletion implicates a role for alphaT-catenin in susceptibility to autism spectrum disorder. J Neurodev Disord 6, 17 (2014).

24. He, Z. et al. Rare-variant extensions of the transmission disequilibrium test: application to autism exome sequence data. Am J Hum Genet 94, 33-46 (2014).

25. Lek, M. et al. Analysis of protein-coding genetic variation in 60,706 humans. Nature 536, 285-291 (2016).

26. Pizzo, L. et al. Rare variants in the genetic background modulate cognitive and developmental phenotypes in individuals carrying disease-associated variants. Genet Med 21, 816-825 (2019). 
27. Pinto, D. et al. Functional impact of global rare copy number variation in autism spectrum disorders. Nature 466, 368-372 (2010).

28. Kanduri, C. et al. The landscape of copy number variations in Finnish families with autism spectrum disorders. Autism Res 9, 9-16 (2016).

29. Yu, T. W. et al. Using whole-exome sequencing to identify inherited causes of autism. Neuron 77, 259-273 (2013)

30. Ionita-Laza, I., Capanu, M., De Rubeis, S., McCallum, K. \& Buxbaum, J. D. Identification of rare causal variants in sequence-based studies: methods and applications to VPS13B, a gene involved in Cohen syndrome and autism. PLoS Genet 10, e1004729 (2014).

31. Kolehmainen, J. et al. Delineation of Cohen syndrome following a large-scale genotype-phenotype screen. Am J Hum Genet 75 , 122-127 (2004)

32. Vernes, S. C. et al. A functional genetic link between distinct developmental language disorders. N Engl J Med 359, 2337-2345 (2008).

33. Toma, C. et al. Comprehensive cross-disorder analyses of CNTNAP2 suggest it is unlikely to be a primary risk gene for psychiatric disorders. PLoS Genet 14, e1007535 (2018).

34. Sebat, J. et al. Strong association of de novo copy number mutations with autism. Science 316, 445-449 (2007).

35. Mikhail, F. M. et al. Clinically relevant single gene or intragenic deletions encompassing critical neurodevelopmental genes in patients with developmental delay, mental retardation, and/or autism spectrum disorders. Am J Med Genet A 155a, 2386-2396 (2011).

36. Voineagu, I. et al. Transcriptomic analysis of autistic brain reveals convergent molecular pathology. Nature 474, 380-384 (2011)

37. Turner, T. N. et al. Genome Sequencing of Autism-Affected Families Reveals Disruption of Putative Noncoding Regulatory DNA. Am J Hum Genet 98, 58-74 (2016)

38. Lionel, A. C. et al. Rare copy number variation discovery and cross-disorder comparisons identify risk genes for ADHD. Sci Transl Med 3, 95 ra75 (2011)

39. Anney, R. et al. A genome-wide scan for common alleles affecting risk for autism. Hum Mol Genet 19, 4072-4082 (2010).

40. Jones, R. M. et al. MACROD2 gene associated with autistic-like traits in a general population sample. Psychiatr Genet 24, 241-248 (2014).

41. Zarrei, M., MacDonald, J. R., Merico, D. \& Scherer, S. W. A copy number variation map of the human genome. Nat Rev Genet 16, 172-183 (2015).

42. van Bon, B. W. et al. Further delineation of the $15 q 13$ microdeletion and duplication syndromes: a clinical spectrum varying from non-pathogenic to a severe outcome. J Med Genet 46, 511-523 (2009).

43. Sharp, A. J. et al. A recurrent 15q13.3 microdeletion syndrome associated with mental retardation and seizures. Nat Genet 40, 322-328 (2008).

44. Rosenfeld, J. A. et al. Deletions flanked by breakpoints 3 and 4 on $15 q 13$ may contribute to abnormal phenotypes. Eur J Hum Genet 19, 547-554 (2011)

45. Okamoto, M. \& Sudhof, T. C. Mints, Munc18-interacting proteins in synaptic vesicle exocytosis. J Biol Chem 272, 31459-31464 (1997).

46. Glessner, J. T. et al. Autism genome-wide copy number variation reveals ubiquitin and neuronal genes. Nature 459, 569-573 (2009).

47. Jarick, I. et al. Genome-wide analysis of rare copy number variations reveals PARK2 as a candidate gene for attention-deficit/ hyperactivity disorder. Mol Psychiatry 19, 115-121 (2014).

48. Yin, C. L. et al. Genome-wide analysis of copy number variations identifies PARK2 as a candidate gene for autism spectrum disorder. Mol Autism 7, 23 (2016).

49. Conceicao, I. C. et al. Definition of a putative pathological region in PARK2 associated with autism spectrum disorder through in silico analysis of its functional structure. Psychiatr Genet 27, 54-61 (2017).

50. Lionel, A. C. et al. Rare exonic deletions implicate the synaptic organizer Gephyrin (GPHN) in risk for autism, schizophrenia and seizures. Hum Mol Genet 22, 2055-2066 (2013).

51. Prasad, A. et al. A discovery resource of rare copy number variations in individuals with autism spectrum disorder. G3 (Bethesda) 2 , $1665-1685$ (2012).

52. Corbett, M. A. et al. A novel X-linked trichothiodystrophy associated with a nonsense mutation in RNF113A. J Med Genet 52, 269-274 (2015)

53. Martins, D., Tavares, I. \& Morgado, C. “Hotheaded”: the role OF TRPV1 in brain functions. Neuropharmacology 85, 151-157 (2014).

54. Han, Q. et al. SHANK3 Deficiency Impairs Heat Hyperalgesia and TRPV1 Signaling in Primary Sensory Neurons. Neuron 92, 1279-1293 (2016).

55. Purcell, S. et al. PLINK: a tool set for whole-genome association and population-based linkage analyses. Am J Hum Genet 81, 559-575 (2007)

56. Raychaudhuri, S. et al. Accurately assessing the risk of schizophrenia conferred by rare copy-number variation affecting genes with brain function. PLoS Genet 6, e1001097 (2010).

57. Chang, C. C. et al. Second-generation PLINK: rising to the challenge of larger and richer datasets. Gigascience 4, 7 (2015).

\section{Acknowledgements}

We gratefully acknowledge all the subjects who have participated in the study. We thank the Centro Interdipartimentale di Ricerche sul Cancro "Giorgio Prodi" (CIRC), University of Bologna for Illumina Sequencing Service. This work was supported by the Italian Ministry of Health (GR-2013-02357561 and RF20091549619) and by University of Bologna (RFO).

\section{Author contributions}

E.B. participated to study design, analyzed and interpreted SNP/CNV data, performed statistical analysis, supervised WES analysis and was a major contributor in writing the manuscript. C.C. performed WES analysis, participated to $\mathrm{CNV}$ analysis and contributed to editing the manuscript and to figures and tables preparation. M.V. performed CNV validation, participated to CNV analysis and contributed to editing the manuscript and to figures and tables preparation. R.I. participated to the sample collection and clinical characterization of patients. A.M. participated to the clinical characterization of patients and to tables preparation. R.T. participated to the sample collection and clinical characterization of patients. A.B. collected the samples, performed the clinical characterization of patients and contributed to review and editing the manuscript. E.M. participated to study design, supervised all analysis and contributed to manuscript writing and revision. All authors read and approved the final manuscript.

\section{Competing interests}

The authors declare no competing interests. 


\section{Additional information}

Supplementary information is available for this paper at https://doi.org/10.1038/s41598-020-59922-3.

Correspondence and requests for materials should be addressed to E.B. or E.M.

Reprints and permissions information is available at www.nature.com/reprints.

Publisher's note Springer Nature remains neutral with regard to jurisdictional claims in published maps and institutional affiliations.

(c) (i) Open Access This article is licensed under a Creative Commons Attribution 4.0 International License, which permits use, sharing, adaptation, distribution and reproduction in any medium or format, as long as you give appropriate credit to the original author(s) and the source, provide a link to the Creative Commons license, and indicate if changes were made. The images or other third party material in this article are included in the article's Creative Commons license, unless indicated otherwise in a credit line to the material. If material is not included in the article's Creative Commons license and your intended use is not permitted by statutory regulation or exceeds the permitted use, you will need to obtain permission directly from the copyright holder. To view a copy of this license, visit http://creativecommons.org/licenses/by/4.0/.

(c) The Author(s) 2020 